\title{
Silkworm breeds and their hybrids of Bombyx mori L. to bmnpv stress
}

\author{
M.H. ASHA AND R.N. BHASKAR \\ Department of Sericulture, University of Agricultural Sciences, G.K.V.K., BENGALURU (KARNATAKA) INDIA
}

BmNPV (Bombyx mori nuclear polyhedrosis virus) causes nuclear polyhedrosis in silkworms. This paper reports on the relative susceptibility of silkworm pure breeds and their hybrids reared under BmNPV stress condition. Infection during fourth and fifth instar silkworm Bombyx mori L., with nuclear polyhedrosis virus caused reduction in larval weight and revealed significant results. However, maximum larval weight of 3.67 and $3.98 \mathrm{~g} / 10$ was noticed in fourth instar inoculated batches $\left(10^{-1}\right.$ and $\left.10^{-3}\right)$ of $\mathrm{CSR}_{2}$. Among hybrids, $\mathrm{CSR}_{4} \mathrm{xCSR}_{16}$ and $\mathrm{CSR}_{2} \mathrm{xCSR}_{4}$ have recorded (5.34 and $\left.5.35 \mathrm{~g} / 10\right)$ and (4.77 and 5.47g/10) compared to other hybrids. On the other hand, fourth instar inoculated batches of fifth instar also recorded maximum larval weight in $\operatorname{CSR}_{2}$ (13.88 and 14.18g/10 and 11.68 and 11.74g/10). Further among hybrids of same instar inoculated, $\mathrm{CSR}_{4} \mathrm{xCSR}{ }_{16}$ recorded (19.06 and $19.90 \mathrm{~g} / 10$ and 20.21 and $\left.21.63 \mathrm{~g} / 10\right)$ which was found maximum than other hybrids. Effective rate of rearing (ERR) of fourth instar inoculated batches were realized differently due to the administration of $B m$ NPV. However, the maximum ERR (59.33 and 64.00\%) and (62.00 and 62.00\%) was recorded in PM which exhibited more survival percentage followed by $\mathrm{CSR}_{4}(58.67$ and $56.00 \%)$ and $(58.67$ and 59.33\%) compare to other two breeds. Among hybrids, PMxCSR 4 was recorded highest ERR (60.67 and 58.00\%) and (58.67 and 57.33\%) when administered with $10^{-1}$ and $10^{-3}$, respectively. The same trend has been noticed even in control lots. The results clearly indicated that, bivoltine breeds and their hybrids reflected low ERR percentage values inturn more sensitive to BmNPV stress.

Key words : Larval weight, ERR, BmNPV, Silkworm breeds

How to cite this paper : Asha, M.H. and Bhaskar, R.N. (2014). Silkworm breeds and their hybrids of Bombyx mori L. to bmnpv stress. Asian J. Bio. Sci., 9 (2) : 220-223. 